\title{
Adaptivity as a Property to Achieve Resilience of Load-Carrying Systems
}

\author{
Schlemmer, Pia D. ${ }^{1, a^{*}}$, Kloberdanz, Hermann ${ }^{1, b}$ \\ Gehb, Christopher M. ${ }^{2, c}$ and Kirchner, Eckhard ${ }^{1, d}$
}

${ }^{1}$ TU Darmstadt, Institute for Product Development and Machine Elements, Otto-Berndt-Str. 2,
64287 Darmstadt, Germany

${ }^{2}$ TU Darmstadt, Institute for System Reliability, Adaptive Structures, and Machine Acoustics SAM, Magdalenenstraße 4, 64289 Darmstadt, Germany

aschlemmer@pmd.tu-darmstadt.de, ${ }^{b}$ kloberdanz@pmd.tu-darmstadt.de, 'gehb@sam.tu-darmstadt.de, ${ }^{d}$ kirchner@pmd.tu-darmstadt.de

Keywords: Resilience, Adaptivity, load-carrying systems, product development

\begin{abstract}
Load-carrying systems often suffer from unexpected disruptions which can cause damages or system breakdowns if they were neglected during product development. In this context, unexpected disruptions summarize unpredictable load conditions, external disturbances or failures of system components and can be comprehended as uncertainties caused by nescience. While robust systems can cope with stochastic uncertainties, uncertainties caused by nescience can be controlled only by resilient load-carrying systems. This paper gives an overview of the characteristics of resilience as well as the time-dependent resilient behaviour of subsystems. Based on this, the adaptivity of subsystems is classified and can be distinguished between autonomous and externally induced adaption and the temporal horizon of adaption. The classification of adaptivity is explained using a simple example of a joint brake application.
\end{abstract}

\section{Introduction - Resilience}

Load-carrying systems in mechanical engineering are often designed to work at a defined design point $d$. In the past years, analyses of several catastrophes have shown that product and system developers must not aim at an optimization of the product regarding a deterministic design point but they have to consider uncertainties. Thus, systems are developed according to the robust design methodology [1, 2]. The Collaborative Research Centre (CRC) 805 "Control of uncertainty in loadcarrying mechanical systems" has made important contributions over the past years. The Robust Design Methodology considers the adjoining area of uncertainty in addition to the specific design point $d$. This area of uncertainty is part of the predefined model's scope and is basically predictable. The considered uncertainty usually is caused by variations of parameters [3]. Systems and products whose behaviour is not affected by this area of uncertainty are called robust [6].

Nevertheless, robust systems sometimes suffer from breakdowns caused by unconsidered and unforeseeable influencing parameters or utilization conditions. This issue can be characterized by uncertainty resulting from nescience and cannot be controlled by robust systems alone.

As soon as the system needs to operate in conditions outside the model's scope, it no longer shows a robust behaviour [7].

Conversely, natural and socio-technical systems are known for surviving and recovering from unexpected impacts or damages. This behaviour and ability is referred to as resilience [8]. In unforeseeable situations, resilient design reduces the impact on the system components, the system environment and the user.

The objective of latest research activities is to control also uncertainties caused by nescience during product development of load-carrying systems by designing systems featuring resilient characteristics. Because a lack of knowledge or information during product development strongly influences the later behaviour of the system, robust design cannot be expected to meet every kind of 
disturbances or component failures in product life time [1,9]. Therefore, developing resilient systems focusses not only on trying to withstand known disturbances but aims at surviving and recovering from unexpected or unforeseeable conditions and influences.

Definition Resilience. The terms resilience and resilient systems are already used in several areas of research. But there is no consistent and generally accepted definition of resilience in engineering science. Additionally, there are different definitions according to the considered application. For example WOODS and HOLLNAGEL define resilience as ,, a paradigm for safety management that focuses on how to help people cope with complexity under pressure to achieve success" [17]. HAIMES (2009) defines resilience as the "(...) ability of the system to withstand a major disruption within acceptable degradation parameters and to recover within an acceptable time and composite costs and risks" [23]. JACKSON'S describes resilience as creating "infrastructure systems that would enable these systems to avoid, withstand, and recover from a broad range of threats" [14].

This paper is based on the definition developed within the CRC 805, as follows:

"A resilient technical system guarantees a predetermined minimum of functional performance even in the event of disturbances or failure of system components, and a subsequent possibility of recovering at least the setpoint function. Resilience can be increased by adjusting the system state via monitoring, responding, learning and/or anticipating, as well as by systematically designing the system topology." [10].

Especially in case of operating outside the model's scope, resilient systems can ensure a defined minimum functionality $f_{\min }$. This ability ensures that the system is not in a dangerous system state or gets seriously damaged by (external) disturbances. Breakdowns of system components and external disturbances are collectively referred to as disruptions.

The resilient behaviour of a system is mainly characterized by the chronological sequence following a disruption [11]. Considering the time-dependent behaviour (see figure 1), both the system response to a disruption (damage phase - phase I) and the behaviour in emergency operation mode (emergency operation phase - phase II) as well as the recovery (recovery phase - phase III) of the system are relevant. In figure $1, f_{\min }$ is the required minimum of functional performance $f$, which represents the total breakdown of the system.

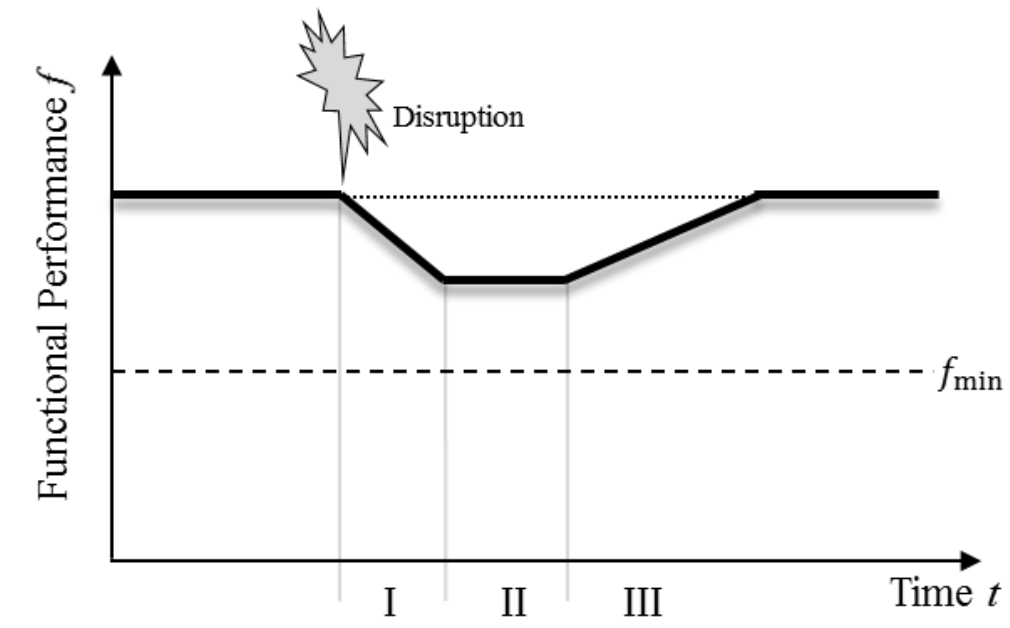

Figure 1. Time-dependent resilient behaviour.

While resilient characteristics are desirable, it must be recognised, that robustness is the prerequisite for resilience [12]. Compared to present design work, the limits of the systems operation beyond the assumed utilization have to be considered additionally (see figure 2). In the context of resilience, it is important to emphasize that the scientific approach to control uncertainty in system design has nevertheless changed considerably.

To illustrate resilient behaviour of technical systems, power grids and truss structures can be looked at as typical examples. The network structure of power grids enables to control load paths in a variety of ways. Truss structures are resilient in a similar way too, by changing load paths in case 
of the failure of one or even more rods. The optimization of truss structures due to resilient characteristics is described in more detail by ALTHERR [10], who emphasizes resilience in mechanical engineering with respect to resilience as a concept for controlling uncertainty during design, production and usage phase of load-carrying structures. Truss structures show a resilient behaviour by changing the load path in case of disturbances or breakdowns of components [10].

\section{Resilience Characteristics and Functions}

The chronological sequence (in figure 1) explains the behaviour of resilient systems in general but does not support the development of resilient systems and subsystems sufficiently. Therefore, resilience functions and static functional resilience characteristics of systems were defined as an outcome of the research work within the CRC 805 as shown in figure 2 and introduced in [10].

Figure 2 shows the static resilient characteristics. The line graph represents the functional performance $f$ of a system depending on influencing factors. The margin $m r$ describes how closely or how critical is currently operating related to any kind of a boundary of the functional performance [7]. The performance range $r$ describes the range of values of the influencing factor $I$ for which the system can ensure a required minimum of functional performance $f_{\min }$. The gracefulness $g$ describes the behaviour of the system near its boundary of the performance range $r$.

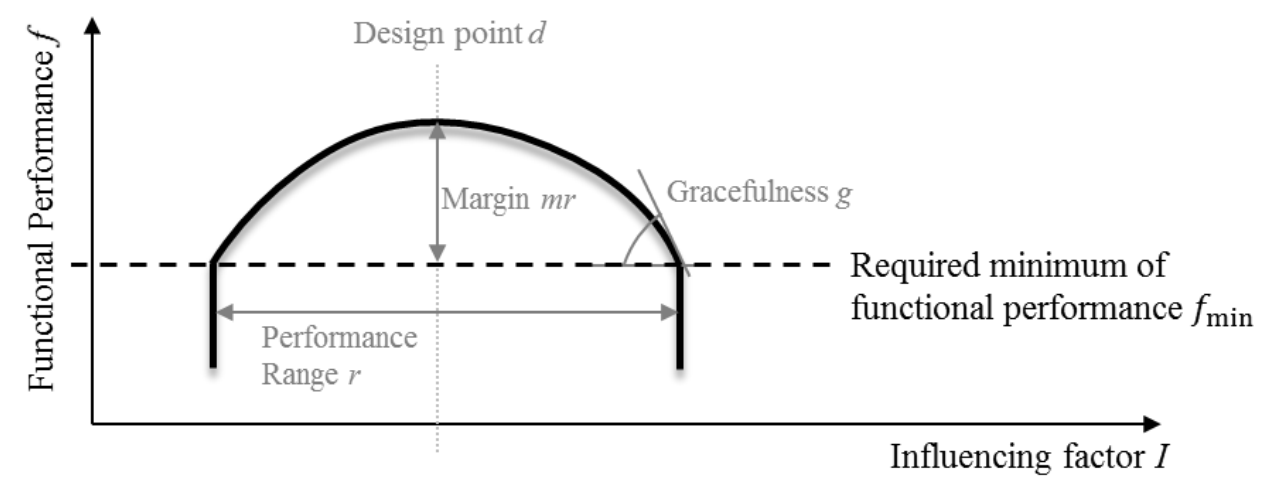

Figure 2. Static resilience characteristics.

These characteristics allow evaluating and comparing systems with respect to their expected resilient behaviour during product development. Measures to optimize resilience characteristics aim at the static resilience characteristics in particular. Therefore, static and time-dependent resilience characteristics are considered both. Figure 3 shows the interdependences between the static and time-dependent resilience characteristics. Before the disruption takes places the functional performance $f$ comply with the functional performance at design point $d$ (phase $t_{1}$ ). In figure $3 \mathrm{~b}$ ), $t_{\mathrm{e}}$ describes the system response to a disruption (damage phase) and $t_{2}$ represents the behaviour in emergency operation mode. The black line shows a system which can recover to its original state after a disruption. The grey line represents the same system after suffering an irreversible damage caused by the disruption.
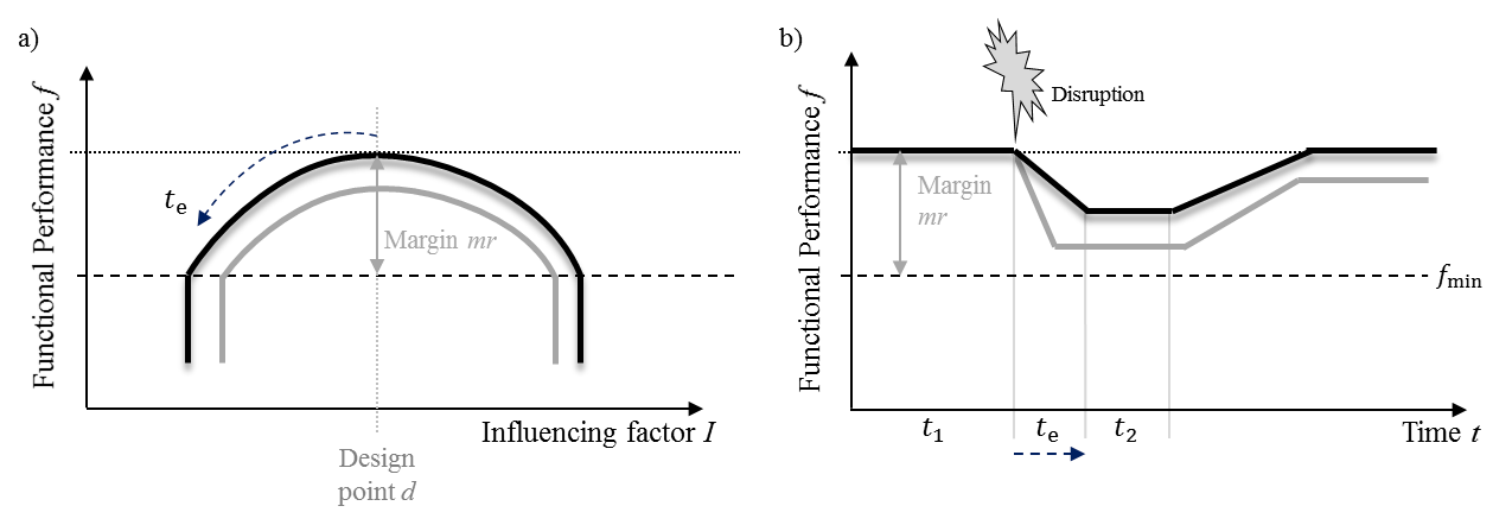

Figure 3. Interdependences between static and time-dependent resilience characteristics. 
The resilience characteristics are basically realized by the resilience functions: monitoring, responding, anticipating and learning. In this context a function is a general intended connection between the input and output of a system that aims for a certain task [13]. Amongst others, this approach corresponds to resilience function descriptions of authors, who are focusing mainly on technical systems [e.g. 7, 8, 14, 15].

Systems need to be able to monitor their environment and their system condition. Monitoring the environment primarily focuses on disturbances which can emerge from the environment (external) and on disruptions caused by component failure within the system (internal). The literature mentions external and systemic disruptions. External disruptions are usually natural disasters like earthquakes or hurricanes [16]. Systemic disruptions are described as "disruptions of function, capability or capacity" [15]. Systemic disruption manifests itself for instance when a component fails [16]. It needs to be emphasized that in addition to disruptions caused by failing components, the interaction between subsystems can also lead to critical situations and disruptions [15].

The resilience function anticipating describes the system's ability to foresee disturbances and opportunities. Changes in environment and risks are anticipated to prevent damages on the system by reacting at an early stage [16; 17]. Resilient systems should also be able to learn from both success and failures. Learning is not possible without continuous monitoring [16].

Furthermore, resilient systems can respond to unforeseen events and impacts. A basic prerequisite for this function is the ability of the system to adapt to its changing environment or conditions. This adaptation can be achieved by “(...) reconfiguration and dynamic reoptimization of available capacities and resources" [16].

Developing resilient systems, the scientific approach is affected significantly by the systems complexity. Especially the design of subsystems with a limited scope differs from the design of large scale network systems.

Complex systems are able to respond flexibly to changing environmental conditions by restructuring of components or by reassignment of functions. Complex systems show a resilient behaviour due to intelligent control capabilities by mechatronic components (e.g. sensors) or due to flexibility usually achieved by human operators [14].

However, this paper does not focus on complex technical systems but on load-carrying subsystems. A subsystem, as shown in figure 4, is a combination of components or assemblies [18]. Subsystems consist mainly of mechanical components or parts and are characterized by a limited scope and restricted functionality. However, this results in a low flexibility of subsystem.

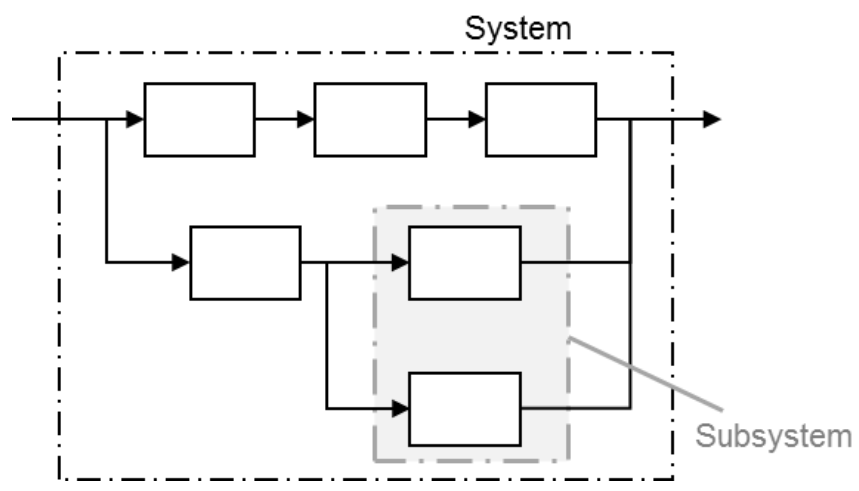

Figure 4. Schematic representation of the subsystem.

Focusing on subsystems is based on the assumption that resilient subsystems can greatly enhance the resilience of the superior system. Furthermore, it is reasonable, since resilient subsystems can be realized without complex signal processing.

Because of the limited complexity, subsystems often do not comprise pronounced signal processing functions and thus they have very diminished ability to learn and to anticipate. Therefore, in the context of resilient subsystems, the functions monitoring and respond are of main interest. 


\section{Adaptivity of Resilient Subsystems}

As mentioned above, resilience of a system can be increased by adjusting the system state. This also applies in general to subsystems. Load-carrying systems and subsystems are able to respond to unexpected disruptions by flexible adjustment [14, 19]. In this context, flexibility describes the fundamental ability of the system to select from different action alternatives [20] e.g. different load paths. The realisation of the resilience function respond of subsystems is achieved by adaptivity; in other words, the system's ability to respond to unpredicted events and influence or to a disruption or even damages is called adaptivity [16].

The adaptivity of load-carrying systems is usually achieved by changing the load path [13]. This ensures, that the load of failed system elements will be carried by other elements to avoid the breakdown of the whole system. In general forces are transferred by mechanical parts. Thus, the realization of the resilience function respond can only be achieved by adapting mechanical parts of the load transmission.

For complex systems, alternative load paths are always available due to their great flexibility, within subsystems, load paths can often not be changed easily. Either there is only one load path facilitated due to their limited flexibility or alternative path cannot be switched because of missing components. The challenge in developing resilient subsystems is therefore to define alternative load paths in advance during product development.

In case of an altered load path, the load transmission is realized by other components. However, these components may not be damaged. So far, redundant components were used to control different load or system conditions [13]. However, redundant components involve additional costs causing a conflict between resilience characteristics and economic efficiency of the system. Applying e.g. principles of functional redundancy can achieve adaptivity without unnecessary additional costs [19].

Adaptivity can be realized either by the system itself (autonomous) or has to be induced externally. This distinction causes a consideration of adaptivity with respect to the temporal horizon of adaptation. Autonomous adaption out of the system itself can mainly be achieved by a spontaneous action. On the other hand, externally induced adaptions are usually realized in mid- or long-term adjustments because an intervention by the operator into the subsystem is required. Externally induced adaptions imply i. a. conversions of the system in general, which take a certain amount of time.

Figure 5 shows the measures by which the adaptivity of mechanical subsystems can be achieved. These measures are explained in more detail below.

\begin{tabular}{|c|c|c|}
\hline \multicolumn{3}{|c|}{ Adaptivity } \\
\hline autonomous & Extern & nduced \\
\hline Short-term & Mid-term & Long-Term \\
\hline $\begin{array}{l}\text { ability to } \\
\text { improvise }\end{array}$ & $\begin{array}{l}\text { Ability to } \\
\text { convert }\end{array}$ & $\begin{array}{l}\text { innovative } \\
\text { capability }\end{array}$ \\
\hline $\begin{array}{l}\text { Physical } \\
\text { redundancy } \\
\text { Functional } \\
\text { redundancy }\end{array}$ & $\begin{array}{l}\text { Replacement } \\
\text { Exchange } \\
\text { Extension }\end{array}$ & $\begin{array}{l}\text { Extension with } \\
\text { development } \\
\text { work }\end{array}$ \\
\hline
\end{tabular}

Figure 5. Measures to achieve adaptivity. 
In load-carrying subsystems, a short-term, autonomous adaptation is effected by a change of the load path, which is already provided by the system itself and usually requires redundant components. In this case, it has to be differentiated between physical redundancy and functional redundancy.

Generally, redundancy means abundance or excess and describes the multiple existence of equal or comparable resources [13]. Redundancy ensures that a breakdown of a certain component of the system is not hazardous in itself, and that redundant resource, can take over its function completely or at least partially [13]. Physical redundancy means, that multiple components with the same function maintain the operation in case of component failure. Functional redundancy means, that the function of the failed component is partly or completely performed by an alternative component with a different function in normal operation. If changing the load path in the subsystem through functional redundancy is possible, resilience characteristics can be achieved with little expense.

Externally induced adaptions may change the load path. However, changing the load path is not compulsory. Externally induced, mid-term adaptions generally describe conversion measures. Subsystems can be converted by replacing, exchanging or extending of parts or components (see figure 9). This differentiation depends on the subsystems functionality after the conversion. Replacement means that the failed component is replaced by exactly the same undamaged component. After replacing the component, the subsystem has the same functionality as before the disruption. An exchange describes surrogating the component by an improved component. In this context, improve means that the system is more robust in relation to disturbances or loads. An exchange leads to an increased functionality of the subsystem. Extension describes a conversion of the subsystem by means of an additional or a greatly improved component, which provides extended or further functionality. All of the conversion measures described above usually require an intervention of a human operator.

Externally induces, long-term adaptions generally characterise the subsystem's innovative capability. The innovative capability describes the subsystem's ability to respond to unexpected disruptions with new adaptation alternatives. This long-term measure require development work.

In order to achieve adaptivity of subsystems, it is important not to focus on single measures but rather to focus on combinations of different measures. For example, the functionality of a subsystem that adapts autonomously in short-term also can be subsequently improved by an exchange (see figure 9).

\section{Example - Joint Brake Application}

In order to clarify the adaptivity of subsystems, a joint brake application is used in this paper. A simple example was purposely chosen to illustrate the effects of adaptivity with respect to resilience. The application is known e.g. from a dental treatment unit. The focus is on moving a device, e.g. a lamp, vertically. Only the movement of the arm downwards is considered in this paper. Figure 6 illustrates the simplified application schematically as well as the basic concept of the joint brake application. 

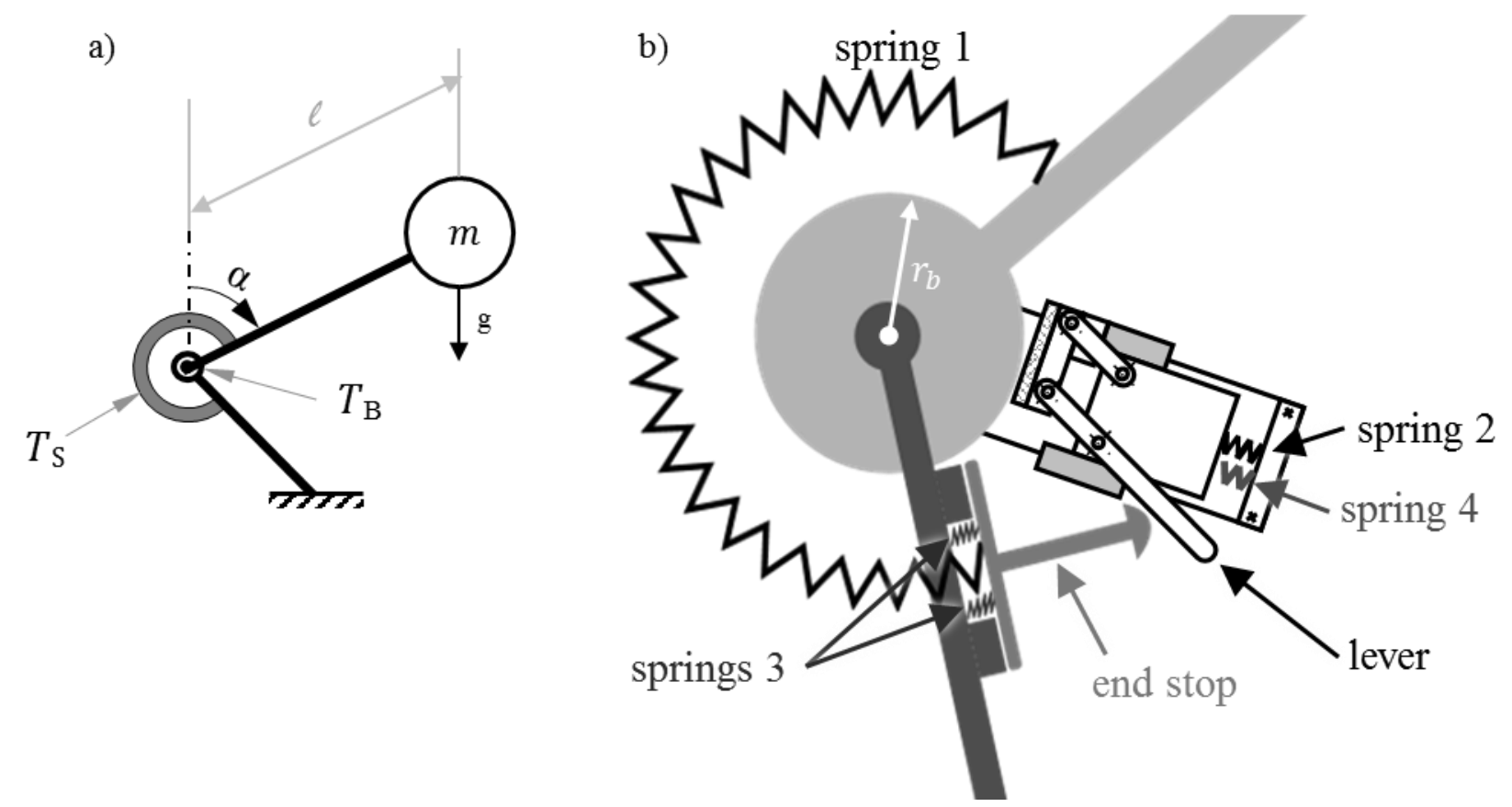

Figure 6. Joint brake application.

The aim of the joint brake application is to hold the mass $m$ in all positions. The mass $m$ causes the torque $T_{\mathrm{m}}$ on the joint.

$$
\begin{gathered}
T_{\mathrm{m}}=m * \mathrm{~g} * \sin \alpha * l \\
T_{\mathrm{m}} \geq T_{\mathrm{B}}+T_{S}
\end{gathered}
$$

A spring (spring 1) and a friction brake in the joint keep the device in place by balancing $T_{\mathrm{m}}$ with the holding torque $T_{\mathrm{h}}$ additional to the spring's and the brake's torque $\left(T_{h}=T_{\mathrm{S}}+T_{\mathrm{B}}\right)$. The spring's and the brake's torque can be compute as follows:

$$
\begin{gathered}
T_{\mathrm{B}}=\mu * F_{S 2} * r_{\mathrm{b}} \\
T_{S}=\mathrm{c} * \alpha
\end{gathered}
$$

This design is robust because it ensures that the holding torque is mostly independent of wear, influence of the environment like temperature, and component tolerances (see figure 8).

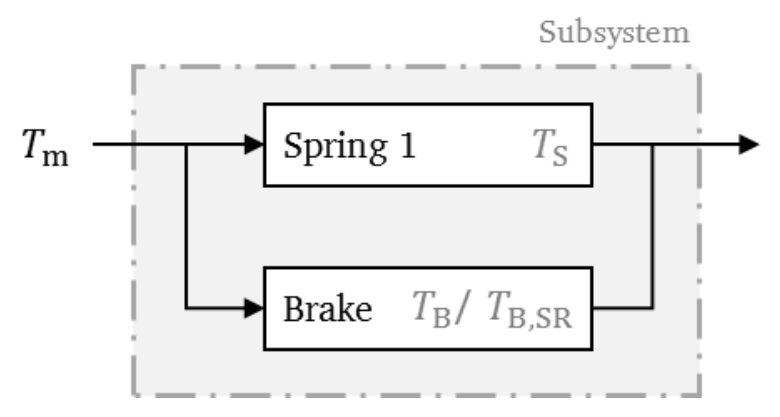

Figure 7. Subsystem of the joint brake application.

The subsystem of the joint brake consists of the brake and spring 1, as shown in figure 7 . The mass $m$ is not part of the subsystem. Therefore, no external influences or additional loads, are known. Only moments acting on the joint as well as the position or angle $(\alpha)$ are known.

Figure 8 shows the course of the torques $T_{\mathrm{m}}, T_{\mathrm{S}}$ and $T_{\mathrm{B}}$ over different angles $\alpha$, whereby the brake's torque $T_{\mathrm{B}}$ dependents on the angular velocity. The curve $T_{\mathrm{m}}$ represents the subsystem behaviour. The system performs as desired if the applied forces and the surrounding conditions are known and within the model's scope. 


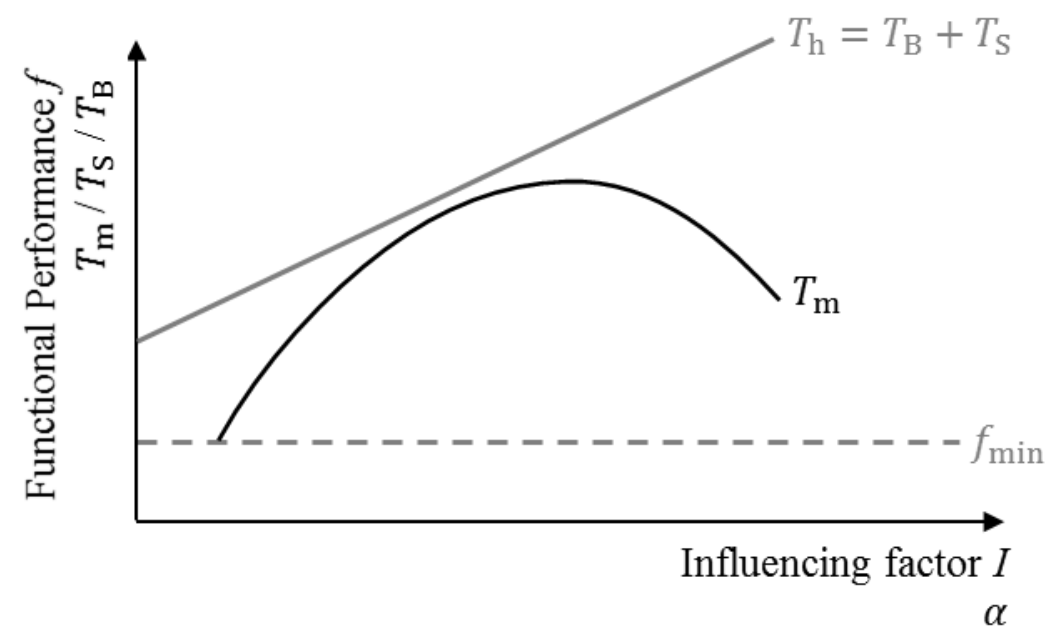

Figure 8. Subsystem behaviour.

Adaptivity of the Joint Brake Application. For the example described above, the adaptivity of the joint brake can be derived. Figure 9 shows the time-dependent behaviour of the joint brake. Functional Performance $f$ represents the load-carrying capacity of the subsystem. Load-carrying capacity describes whether and how far the mass $m$ can be held by the joint brake.

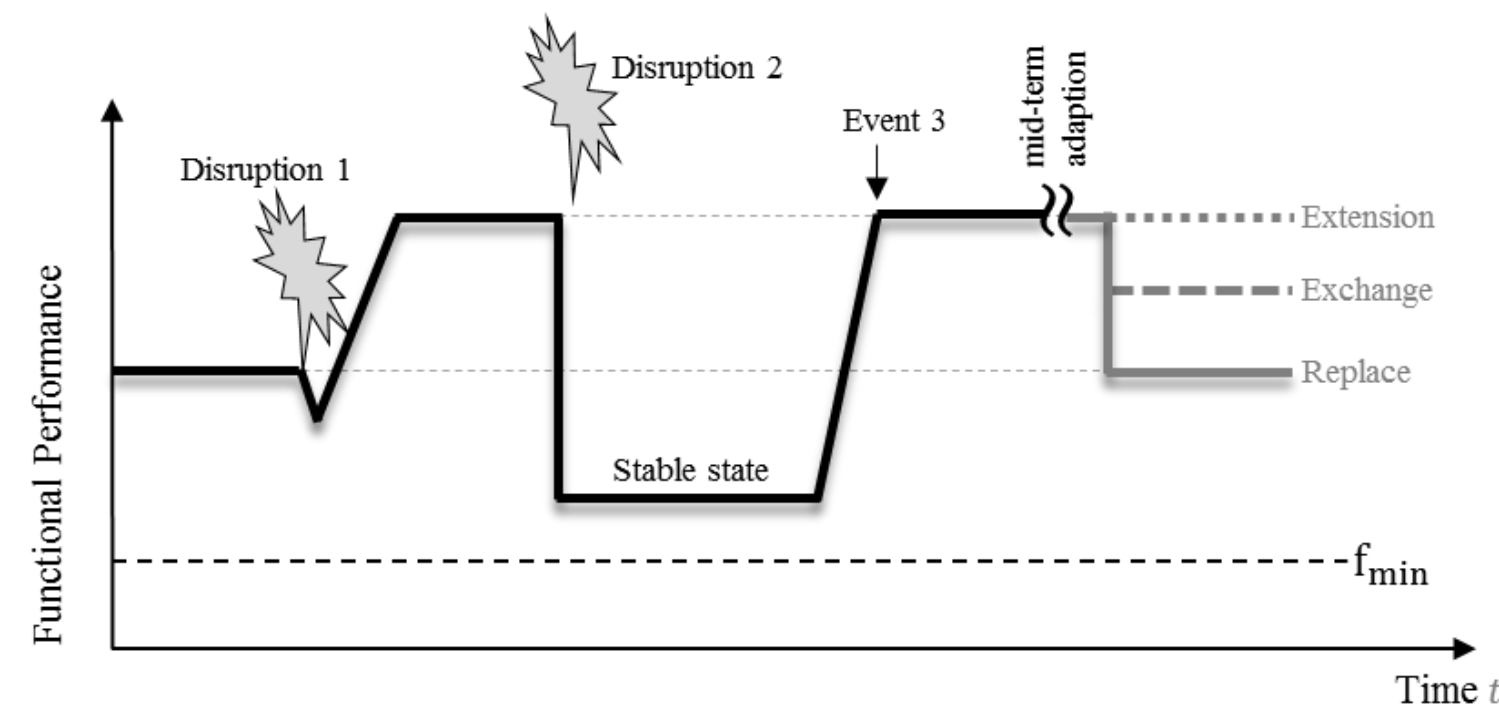

Figure 9. Time-dependent behaviour of the joint brake.

Disruption 1 describes the case of a component failure (internal disruption), in this context the tearing of spring 1 . If spring 1 breaks, the spring's torque $T_{\mathrm{S}}$ abolished, leaving only the brake's torque $T_{\mathrm{B}}$. As a result, the brake holds the entire mass $m$ alone. However, the brake's torque $T_{\mathrm{B}}$ is actually smaller than the torque $T_{\mathrm{m}}$, which results from the mass. Therefore, the mass $m$ cannot be held for the first moment, and the functional performance decreases (see figure 9).

When spring 1 breaks, springs 3 push the lever through the end stop, so that the brake is in a self-reinforcing state. This increases the torque applied by the self-reinforced brake $\left(T_{\mathrm{BSr}}\right)$ [4]. Since the brake is quickly pushed into the self-reinforcing state, the decrease of the functional performance is marginal (see figure 9). This short-term adaption occurs within the system itself (autonomously).

If an additional external load in the form on an overload is added to the broken spring 1 (disruption 2), the brake's self-reinforcement state is switched into a state of self-destruction [4]. This self-destruction must be prevented by resilient behaviour of the subsystem. Therefore, in addition to the principle of self-reinforcement, the principle of Bi-Stability is also necessary. The brake is brought into a further stable state by spring 4 and the self-destruction of the brake can be prevented. 
As soon as the overload is no longer there (event 3), the subsystem returns to self-reinforcing level (see figure 9). In mid-term, the subsystem can only perform again at the normal level by convert the subsystem by replacing, exchanging or extending the spring.

It is possible to replace spring 1 (case 1). In this case, the functionality of the joint brake is the same as in the initial state. However, the spring can also be replaced. Here an improved spring is installed. This leads to improved functionality of the system.

In the long term, an extension can also take place, whereby an additional element - for example a viscous damper - can be integrated in the joint brake. This case is not illustrated in figure 9 .

\section{Example - Guidance Elements with Friction Brake}

If predefined load paths in a load-carrying structure are not suitable anymore because of fatigue or damage, load redistribution during usage can be an option to prevent further harm or reduce severity of damage. Using friction brakes in a kinematic links' joint is a new approach to achieve that goal and demonstrate the adaptation/adaptivity which is realized with the help of mechatronic semi-active components.

The following example describes a test rig to investigate the ability of load redistribution during operation due to semi-active structure manipulation, [21] and [22], Fig. 10a). The test rig is derived from a complex load-bearing system that is currently developed in the CRC 805. This load-bearing system is a modular active spring damper system (German acronym MAFDS) and serves as an example for a modular structure with passive, semi-active and active modules to study uncertainty in different approaches to control stability and vibration as well as redistribute load paths, [5].

The basic idea is to shift loads that primarily only go through the spring-damper 7 also go through the semi-active controlled guidance elements 1 or 2, depending on which support 3 or 4 is damaged. Load redistribution means shifting load between the supports 3 and 4 during operation according to current or anticipated damage, Fig. 1(b). The guidance elements are equipped with electromagnetic friction brakes 10 and 11 at the middle joints. Within the friction brakes, the semiactive normal force acting on the friction lining is controlled and induced by the brake's electromagnet. It causes a torque and can change the load transmitting properties of the guidance element by increasing or decreasing the friction force in the joint.

a)

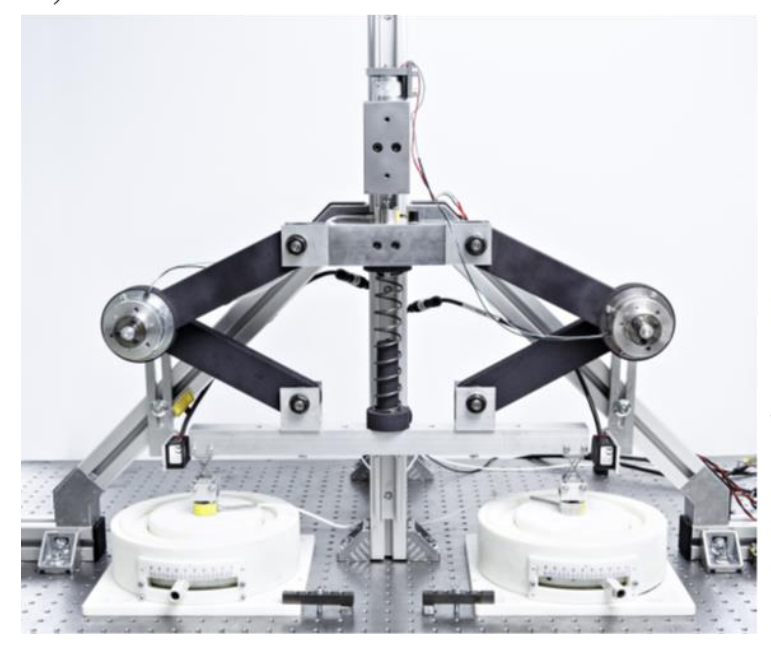

b)

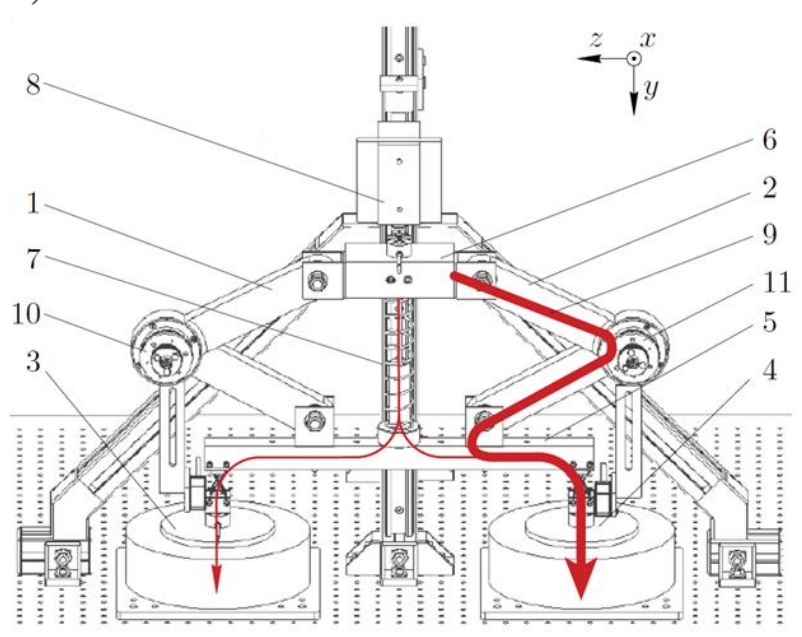

Fiure 10. a) semi-active load redistribution test rig and b) schematic load path within the structure.

If support 3 is assumed to be damaged or weak, it can be relieved by using the semi-active augmented guidance element 2 as an additional load path 9 to the spring-damper 7 , so that parts of the load can be bypassed through the guidance element 2 directly to the undamaged support 4 . The additional load path 9 is highlighted in Fig. 1(b) by a solid red line passing through the right guidance element 2 . Without applying any additional friction force due to the brakes, the guidance elements 1 and 2 fulfil only kinematic tasks. Their main function is to enable a defined up-and- 
down compression stroke trajectory of the connected structure parts beam 5 and mass 6 . The semiactive guidance element become kinetic when they generate friction forces and, respectively, torques in the middle joints.

\section{Conclusions and Outlook}

In this paper, the adaptivity of subsystems was classified. Adaptivity can be distinguished based on the temporal horizon of adaptivity. With respect to the temporal distinction, short-term, medterm and long-term adjustments can be distinguished. All of them require continuous monitoring by the system itself. Short-term adjustments also called reaction usually occur out of self-help. Shortterm adjustments with external help are usually harder to realize. Further it can be classify by the degree of adaptivity. Degree of adaptivity describes the external influence with respect to the adaption. For example mechanical systems can adapt their environment through replacing/exchanging components or through adding further components. Thereby it should be possible to add components without changing the component's topology.

Subsystems fulfil two of the four known resilience functions: monitoring, reacting, anticipating, and learning, namely: monitoring and reacting. At the subsystem level anticipation is only possible with an additional signal. For example this signal can be provided from the complex systems. The adaptivity of subsystems with the help of another signal will be researched by the authors.

\section{Acknowledgement}

Collaborative Research Centre (CRC) 805 "Control of uncertainty in load-carrying mechanical systems" achieved the results presented here in subproject "A1: Development of Models, Methods and Instruments for the Acquisition, Description and Evaluation of Uncertainties". Thank you to the Deutsche Forschungsgemeinschaft (DFG) for funding and supporting CRC 805.

\section{References}

[1] H. Hanselka, R. Platz, Ansätze und Maßnahmen zur Beherrschung von Unsicherheit in lasttragenden Systemen des Maschinenbaus, In Konstruktion. November/Dezember 2010, S. 55-62.

[2] T. Freund, Konstruktionshinweise zur Beherrschung von Unsicherheit in technischen Systemen, Dissertation, Technische Universität Darmstadt, Fachgebiet für Produktentwicklung und Maschinenelemente, 2018.

[3] T. Eifler, Modellgestützte Methodik zur systematischen Analyse von Unsicherheit im Lebenslauf technischer Systeme, Dissertation, Technische Universität Darmstadt, Fachgebiet für Produktentwicklung und Maschinenelemente, 2014.

[4] R. Kühnpast, Das System der selbsthelfenden Lösungen in der maschinenbaulichen Konstruktion, Dissertation, Technische Hochschule Darmstadt, Fachgebiet für Maschinenelemente, 1968.

[5] S. Mallapur and R. Platz, "Quantification and evaluation of uncertainty in the mathematical modelling of a suspension strut using bayesian model validation approach," in Model Validation and Uncertainty Quantification, Volume 3 (Barthorpe Robert J., R. Platz, I. Lopez, B. Moaveni, and C. Papadimitriou, eds.), Conference proceedings of the Society for Experimental Mechanics series, (Cham), pp. 113\{124, Springer International Publishing, 2017.

[6] T. Eifler, G. C. Enss, M. Haydn, L. Mosch, R. Platz, H. Hanselka, Approach for a Consistent Description of Uncertainty in Process Chains of Load Carrying Mechanical Systems. In: Applied Mechanics and Materials. Vol. 104, pp. 133-144. (2011) Trans Tech Publications. DOI: 10.4028/www.scientific.net/AMM.104.133. 
[7] D. D. Woods, Essential Characteristics of Resilience, in: E. Hollnagel, D. Woods and N. Leveson, (Ed.), Resilience Engineering - Concepts and Precepts, Ashgate, transferred to digital printing in 2010, Farnham, pp. 21-34.

[8] E. Hollnagel and D. D. Woods, Epilogue: Resilience Engineering Precepts, in: E. Hollnagel, D. D. Woods and N. Leveson (Ed.), Resilience Engineering - Concepts and Precepts, Ashgate, transferred to digital printing in 2010, Farnham, pp. 347-358.

[9] J. Würtenberger, S. Gramlich, T. Freund, J. Lotz, M. Zocholl, H. Kloberdanz, Uncertainty in product modelling within the development process, In Applied Mechanics and Materials. Vol. 807, pp. 89-98, Trans Tech Publications. DOI: 10.4028/www.scientific.net/AMM.807.89

[10] L. Altherr, N. Brötz, I. Dietrich, T. Gally, F. Geßner, H. Kloberdanz, P. Leise, P. Schlemmer and A. Schmitt, Resilience in Mechanical Engineering - A Concept for Controlling Uncertainty during Design, Production and Usage Phase of Load-Carrying Structures, In Applied Mechanics and Materials. Trans Tech Publications. Under review.

[11] K. Tierney and M. Bruneau, Conceptualizing and measuring resilience: A key to disaster loss reduction, TR News 250 (2007).

[12] J. Mathias, H. Kloberdanz, R. Engelhardt and H. Birkhofer, Strategies and Principles to design robust products, Proceedings of the DESIGN 2010 / 11th International Design Conference, Dubrovnik, Croatia, May 17-20, 2010, pp. 341-350.

[13] Pahl, G., Beitz, W., Feldhusen, J. and Grote K. H. (2007), Engineering Design: A Systematic Approach, third Edition, Springer London.

[14] S. Jackson, Evaluation of Resilience Principles for Engineered Systems, PhD thesis, University of South Australia, 2016.

[15] S. Jackson, Architecting Resilient Systems: Accident Avoidance and Survival and Recovery from Disruptions, Wiley, Hoboken, 2009.

[16] A. M. Madni and S. Jackson, Towards a Conceptual Framework for Resilience Engineering, IEEE Systems Journal, July 2009, DOI: 10.1109/JSYST.2009.2017397

[17] D. D. Woods and E. Hollnagel, Prologue: Resilience Engineering Concepts, in: E. Hollnagel, D. D. Woods and N. Leveson, (Ed.), Resilience Engineering - Concepts and Precepts, Ashgate, transferred to digital printing in 2010, Farnham, pp. 1-6.

[18] J. Feldhusen, K.-H. Grote, G. Pahl, W. Beitz, eds., Pahl/Beitz Konstruktionslehre: Methoden und Anwendung erfolgreicher Produktentwicklung. 8. vollst. überarb. Aufl., Springer Verlag, Berlin, Heidelberg 2013.

[19] Z. Sun, G.S. Yang, B. Zhang and W. Zhang, On the Concept of Resilient Machine. 6th IEEE Conference on Industrial Electronics and Applications 2011, 978-1-4244-8756-1/11

[20] H. Meffert, Zum Problem der betriebswirtschaftlichen Flexibilität. Zeitschrift für Betriebswirtschaft, 1969, vol. 39, no. 12, pp. 779-800.

[21] C. M. Gehb, R. Platz, and T. Melz, Active load path adaption in a simple kinematic loadbearing structure due to stiffness change in the structure's supports, Journal of Physics: Conference Series, vol. 744, no. 1, p. 012168, 2016.

[22] C. M. Gehb, R. Platz, and T. Melz, Global load path adaption in a simple kinematic loadbearing structure to compensate uncertainty of misalignment due to changing stiffness conditions of the structure's supports, in: Model Validation and Uncertainty Quantification, Volume 3 (Barthorpe Robert J., R. Platz, I. Lopez, B. Moaveni, and C. Papadimitriou, eds.), Conference proceedings of the Society for Experimental Mechanics series, (Cham), pp. 133-144, Springer International Publishing, 2017.

[23] Y. Y. Haimes, Y. Y., On the Definition of Resilience in Systems. Risk Analysis, 2009, Vol. 29, No. 4, pp. 498-501. 